\title{
Д.А. Иванычев
}

\section{МЕТОД ГРАНИЧНЫХ СОСТОЯНИЙ В РЕШЕНИИ ПЕРВОЙ ОСНОВНОЙ ЗАДАЧИ ТЕОРИИ АНИЗОТРОПНОЙ УПРУГОСТИ С МАССОВЫМИ СИЛАМИ ${ }^{1}$}

\begin{abstract}
Представлена методика определения напряженно-деформированного состояния анизотропных тел вращения от одновременного действия поверхностных и массовых сил. Теория представляет собой развитие метода граничных состояний. Особенность решения состоит в том, что след искомого упругого поля удовлетворяет одновременно поверхностным и массовым силам, а не представляет собой сумму решений частных задач.
\end{abstract}

Ключевые слова: метод граничных состояний, анизотропия, массовые силы, краевые задачи, пространство состояний, первая основная задача.

Детали из современных материалов, такие, как эластомеры, поликристаллические металлы, керамика, а также композитные материалы, обладающие значительной анизотропией свойств, применяемые в конструкциях, механизмах и машинах, часто пребывают в сложных условиях нагружения. На тело, находящееся в таких условиях, действуют поверхностные и массовые силы. Определение напряженно-деформированного состояния тел от совокупности таких воздействий, а также в силу анизотропии упругих свойств материала, составляет актуальную научную задачу.

Массовые силы рассматривались в работах различного направления механики. Например, в работе [1] установлена зависимость между критическим состоянием твердого тела, предшествующим разрушению, и взаимным влиянием угловых и линейных деформаций друг на друга, что сопровождается высвобождением их внутренней энергии. В работе [2] представлено численно-аналитическое решение плоской задачи теории упругости с использованием метода взвешенных невязок в форме метода граничного решения. Найдены распределения напряжений и смещений в упругом теле, подверженном действию заданной системы объемных сил и заданных напряжений или смещений на границах. Авторами [3] рассмотрена задача о напряженно-деформированном состоянии твердого тела с учетом ползучести и находящегося под действием объемных сил. В [4] исследовались вынужденные деформации в виде суммы воздействий поверхностных и объемных сил. В работе [5] с использованием фиктивных расчетные схем, основанных на эквивалентности воздействий в механике деформируемого твердого тела, получены напряженно-деформированные состояния для балки на двух опорах, находящейся под действием массовых сил; вращающегося тонкого круглого диска; плотины треугольного поперечного сечения, находящейся под действием объемных фильтрационных сил. В работе [6] представлены задачи теории упругости с заданными объемными и поверхностными силами в функциональных энергетиче-

\footnotetext{
${ }^{1}$ Исследование выполнено при финансовой поддержке РФФИ и Липецкой области в рамках научного проекта № 19-41-480003 "p_a".
} 
ских пространствах тензоров напряжений и деформаций; методом ортогональных проекций решены конкретные задачи. Объемные силы рассматривались и в механике разрушения [7]: дается решение задачи механики разрушения о зарождении трещин в металлическом круговом диске под действием объемных сил. Авторами [8] для перемещений получено условие эквивалентности поверхностных и объемных сил с использованием вариационного уравнения Лагранжа. Оценке вклада гравитационных сил в возмущение напряженно-деформированного состояния упругого полупространства, вызванное шаровой неоднородностью упругих свойств, расположенной на некоторой глубине посвящена работа [9]. В [10] приведено построение поля перемещений для изотропного упругого тела, ограниченного концентрическими сферами и находящегося под действием осесимметричных нестационарных объемных сил. В работе [11] показано влияние силы тяжести на деформированное состояние идеальнопластического пространства, ослабленного цилиндрической полостью, а в [12] получены точные аналитические решения задач о равновесии толстостенных трансверсально-изотропных составных сфер с жестко закрепленной границей и находящихся под действием массовых сил и внутреннего давления. Для анизотропного стержня с одной плоскостью упругой симметрии решена задача кручения с помощью метода граничных состояний [13].

Обратный метод определения напряженно-деформированного состояния изотропных упругих тел от действия непрерывных непотенциальных массовых сил приведен в работе [14].

Метод граничных состояний с участием объемных сил для изотропной среды применен авторами [15]. В [16] разработана методика получения полнопараметрических решений для анизотропных тел, где возникновение фиктивных массовых сил являлось следствием применения метода Пуанкаре.

Метод граничных состояний относится к разряду энергетических методов. С помощью метода минимизации полной энергии деформации, решена задача по определению напряженно-деформированного состояния, возникающего при осадке жесткопластической тонкой квадратной заготовки [17]. В работе [18] в осесимметричной постановке с помощью непрямого метода граничных состояний показано решение уравнения Лапласа, а в [19] рассмотрены задачи контакта без трения трансверсально-изотропного цилиндра в условиях одновременного действия массовых и поверхностных сил.

В рамках настоящей работы предполагается развитие энергетического метода граничных состояний на класс краевых задач теории упругости с массовыми силами для трансверсально-изотропных тел вращения. Особенность искомого упругого поля состоит в том, что его след одновременно удовлетворяет заданным условиям на границе и внутри области, т.е. массовым силам, а не представляет собой сумму отдельных полей в задаче эластостатики и в задаче определения напряженно-деформированного состояния от действия массовых сил.

\section{1. Постановка задачи}

Рассматривается равновесие трансверсально-изотропного тела, ограниченного одной или несколькими коаксиальными поверхностями вращения под действием осесимметричных (не зависящих от угла $\theta$ в цилиндрической системе координат $r, Q, z)$ поверхностных усилий $\mathbf{p}_{\mathbf{v}}=\left\{p_{r}, p_{z}\right\}$ и массовых сил $\mathbf{X}=\{R, Z\}$, симметрично распределенных относительно оси вращения z (рис. 1). 


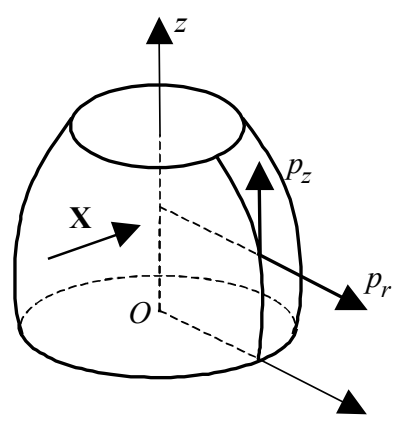

Рис. 1. Трансверсально-изотропное тело вращения

Fig. 1. A transversely isotropic body of revolution

Решение поставленной задачи может быть проведено простым путем: сначала решить краевую осесимметричную задачу механики от внешних сил, приложенных к поверхности тела, затем отдельно решить задачу по определению упругого состояния от массовых сил, а полученные упругие поля сложить. Однако в этом случае сложно проводить анализ полученного результата исходя из теорий прочности и жесткости. Возникает необходимость дискретно корректировать граничные условия в краевой задаче, что составляет непростую и трудоемкую задачу, особенно если граница тела частично или полностью защемлена. Например, естественно, что напряжения внутри тела, находящегося под действием сил инерции со свободной границей, отличаются от напряжений в том же теле с защемленной границей, вопрос состоит в том, каким образом происходит это перераспределение.

Целью работы является развитие метода граничных состояний на класс краевых осесимметричных задач теории упругости для анизотропных тел, в которых упругое поле от массовых сил не просто суммируется с полем от внешней уравновешенной нагрузки, а входит в состав решения, позволяя получить то самое перераспределение напряжений от влияния граничных условий. Ее достижению отвечает система взаимосвязанных процедур: корректная постановка, обезразмеривание, выбор метода решения с построением определяющей теории, решение конкретных задач.

\section{2. Определяющие соотношения}

В случае осесимметричной деформации тела вращения точки перемещаются лишь в меридианных плоскостях и компоненты напряженно-деформированного состояния не зависят от угла $\theta$, т.е. напряжения $\tau_{r \theta}, \tau_{z \theta}$ и деформации $\gamma_{r \theta}, \gamma_{z \theta}$ равны нулю.

Для однородного трансверсально-изотропного упругого тела в цилиндрических координатах имеют место следующие соотношения [20].

Дифференциальные уравнения равновесия:

$$
\begin{gathered}
\frac{\partial \tau_{z r}}{\partial z}+\frac{\partial \sigma_{r}}{\partial r}+\frac{\sigma_{r}-\sigma_{\theta}}{r}+R=0 ; \\
\frac{\partial \sigma_{z}}{\partial z}+\frac{\partial \tau_{z r}}{\partial r}+\frac{\tau_{z r}}{r}+Z=0 .
\end{gathered}
$$


Соотношения Коши:

$$
\varepsilon_{z}=\frac{\partial w}{\partial z} ; \varepsilon_{r}=\frac{\partial u}{\partial r} ; \varepsilon_{\theta}=\frac{u}{r} ; \gamma_{z r}=\frac{\partial w}{\partial r}+\frac{\partial u}{\partial z}
$$

Обобщенный закон Гука:

$$
\begin{gathered}
\varepsilon_{z}=\frac{1}{E_{z}}\left[\sigma_{z}-v_{z}\left(\sigma_{r}+\sigma_{\theta}\right)\right] ; \\
\varepsilon_{r}=\frac{1}{E_{r}}\left(\sigma_{r}-v_{r} \sigma_{\theta}\right)-\frac{v_{z}}{E_{z}} \sigma_{z} ; \\
\varepsilon_{\theta}=\frac{1}{E_{r}}\left(\sigma_{\theta}-v_{r} \sigma_{r}\right)-\frac{v_{z}}{E_{z}} \sigma_{z} ; \gamma_{z r}=\frac{1}{G_{z}} \tau_{z r} .
\end{gathered}
$$

Здесь $u, w$ - компоненты вектора перемещений $\mathbf{u}$ вдоль оси $r$ и $z$ соответственно; $\varepsilon_{r}, \varepsilon_{\theta}, \varepsilon_{z}, \gamma_{z r}$ - компоненты тензора деформаций; $\sigma_{r}, \sigma_{\theta}, \sigma_{z}, \tau_{z r}$ - компоненты тензора напряжений; $R, Z$ - компоненты вектора массовых сил $\mathbf{X}$ вдоль соответствующей оси; $E_{z}$ и $E_{r}-$ модули упругости соответственно в направлении оси $z$ и в плоскости изотропии; $v_{z}$ - коэффициент Пуассона, характеризующий сжатие вдоль оси $r$ при растяжении вдоль оси $z ; v_{r}$ - коэффициент Пуассона, характеризующий поперечное сжатие в плоскости изотропии при растяжении в этой же плоскости; $G_{r}$ и $G_{z}$ - модули сдвига в плоскости изотропии и перпендикулярной к ней.

\section{3. Метод решения}

Для решения первой основной задачи теории упругости прибегнем к понятиям метода граничных состояний (МГС) [21]. МГС является энергетическим методом решения задач уравнений математической физики. Он показал свою эффективность в решении краевых задач теории упругости, как для изотропных, так и для анизотропных сред, в решении задач термоупругости, гидродинамики идеальной жидкости, динамики (колебаний) изотропных тел и др.

Основу метода составляют пространства внутренних $\Xi$ и граничных $\Gamma$ состояний:

$$
\Xi=\left\{\xi_{1}, \xi_{2}, \xi_{3}, \ldots, \xi_{k}, \ldots\right\} ; \Gamma=\left\{\gamma_{1}, \gamma_{2}, \gamma_{3}, \ldots, \gamma_{k}, \ldots\right\} .
$$

Внутреннее состояние определяется наборами компонент вектора перемещений, тензоров деформаций и напряжений:

$$
\xi_{k}=\left\{u_{i}^{k}, \varepsilon_{i j}^{k}, \sigma_{i j}^{k}\right\} .
$$

Воспользуемся при построении решения основных задач механики уравнением Клапейрона [22], [23]:

$$
\int_{V} \mathbf{X} \mathbf{u} d V+\int_{S} \mathbf{p} \mathbf{u}_{\mathbf{v}} d S-\int_{V} \sigma_{i j} \varepsilon_{i j} d V=0
$$

где $\mathbf{p}$ и $\mathbf{u}_{\mathbf{v}}$ - векторы поверхностных усилий и перемещения точек границы.

Скалярное произведение в пространстве $\Xi$ внутренних состояний выражается через внутреннюю энергию упругого деформирования (отсюда и принадлежность 
метода к классу энергетических). Например, для 1-го и 2-го внутренних состояний тела, занимающего область $V$ :

$$
\left(\xi_{1}, \xi_{2}\right)=\int_{V} \varepsilon_{i j}^{1} \sigma_{i j}^{2} d V
$$

причем в силу тождества Бетти:

$$
\left(\xi_{1}, \xi_{2}\right)=\left(\xi_{2}, \xi_{1}\right)=\int_{V} \varepsilon_{i j}^{1} \sigma_{i j}^{2} d V=\int_{V} \varepsilon_{i j}^{2} \sigma_{i j}^{1} d V .
$$

Граничное состояние $\gamma_{k}$, в отличие от традиционного $\gamma_{k}=\left\{u_{v i}^{k}, p_{i}^{k}\right\}$, определяемого в [21], будем формировать наборами компонент вектора перемещения точек границы $u_{v i}$, поверхностными усилиями $p_{i}$ и массовыми силами $X_{i}$ ( $X_{1}=R, X_{2}=Z$ ). Последнее условно в силу того, что массовые силы не относятся к элементу поверхности тела:

$$
\gamma_{k}=\left\{u_{v i}^{k}, p_{i}^{k}, X_{i}^{k}\right\} ; p_{i}^{k}=\sigma_{i j}^{k} n_{j} ; i, j=1,2,
$$

где $n_{j}$ - компонента нормали к границе.

В пространстве граничных состояний $Г$ согласно (6) скалярное произведение выражает работу внешних сил по поверхности тела $S$ и работу массовых сил на перемещениях $u_{i}$ внутренних точек тела, например для 1-го и 2-го состояний:

$$
\left(\gamma_{1}, \gamma_{2}\right)=\int_{S} p_{i}^{1} u_{v i}^{2} d S+\int_{V} X_{i}^{1} u_{i}^{2} d V
$$

причем в силу тождества Бетти и соотношения Клапейрона

$$
\left(\gamma_{1}, \gamma_{2}\right)=\left(\gamma_{2}, \gamma_{1}\right)=\int_{S} p_{i}^{1} u_{v i}^{2} d S+\int_{V} X_{i}^{1} u_{i}^{2} d V=\int_{S} p_{i}^{2} u_{v i}^{1} d S+\int_{V} X_{i}^{2} u_{i}^{1} d V
$$

В случае гладкой границы и в силу единственности решения задач линейной теории упругости оба пространства состояний являются гильбертовыми и сопряжены изоморфизмом. Каждому элементу $\xi_{k} \in \Xi$ соответствует единственный элемент $\gamma_{k} \in \Gamma$, причем это соответствие взаимно-однозначное: $\xi_{k} \leftrightarrow \gamma_{k}$. Это позволяет отыскание внутреннего состояния свести к построению изоморфного ему граничного состояния.

Основную сложность формирования решения в МГС составляет конструирование базиса внутренних состояний, который опирается на общее или фундаментальное решение для среды; также возможно использование каких-либо частных или специальных решений. Здесь предлагается несколько иная методика конструирования базиса внутренних состояний, которая изложена ниже.

Для формирования определяющих соотношений базисы пространств состояний необходимо проортонормировать. Ортонормирование базиса пространства $\Xi$ осуществляется по разработанному рекурсивно-матричному алгоритму ортогонализации [24], где в качестве перекрестных скалярных произведений принимается (7). Алгоритм основан на процессе Грама - Шмидта, переписанном в форме, использующей лишь перекрестные скалярные произведения элементов исходного базиса, которые сведены в матрицу Грама. Если в процессе ортогонализации на $k$-м шаге встречается некоторый элемент базиса внутренних состояний $\xi_{k}$, алго- 
ритм на этом шаге выдаст 0 (нулевое), если этот элемент является линейной комбинацией элементов $\xi_{1}, \ldots, \xi_{k-1}$. Для сохранения ортогональности выходных элементов и для предотвращения деления на ноль при ортонормировании алгоритм делает проверку на нулевые элементы и исключает их. На их место идут следующие элементы исходного базиса внутренних состояний и процесс повторяется.

Ортонормированный базис $\Gamma$ редуцируется из ортонормированного базиса внутренних состояний при использовании (8) и (1).

Окончательно, проблема сводится к разрешающей системе уравнений относительно коэффициентов Фурье, разложения искомых внутреннего $\xi$ и граничного $\gamma$ состояний в ряд по элементам ортонормированного базиса:

$$
\xi=\sum_{k=1}^{\infty} c_{k} \xi_{k} ; \gamma=\sum_{k=1}^{\infty} c_{k} \gamma_{k}
$$

или в развернутом виде

$$
p_{i}=\sum_{k=1}^{\infty} c_{k} p_{i}^{k} ; u_{i}=\sum_{k=1}^{\infty} c_{k} u_{i}^{k} ; \sigma_{i j}=\sum_{k=1}^{\infty} c_{k} \sigma_{i j}^{k} ; \varepsilon_{i j}=\sum_{k=1}^{\infty} c_{k} \varepsilon_{i j}^{k} ; X_{i}=\sum_{k=1}^{\infty} c_{k} X_{i}^{k} .
$$

В случае первой основной задачи, где заданы массовые силы $\mathbf{X}=\{R, Z\}$ и усилия на границе $\mathbf{p}=\left\{p_{r}, p_{z}\right\}$, коэффициенты Фурье $c_{k}$ определяются из выражения

$$
c_{k}=\int_{V} \mathbf{X} \mathbf{u}^{k} d V+\int_{S} \mathbf{p} \mathbf{u}_{\mathbf{v}}^{k} d S
$$

где $\mathbf{u}^{k}$ и $\mathbf{u}_{\mathbf{v}}^{k}-$ вектор перемещения в $k$-м базисном элементе базиса внутренних (5) и граничных (8) состояниях соответственно.

Тестирование коэффициентов Фурье осуществляется подстановкой одного из базисных элементов с соответствующими ГУ в качестве заданного, при этом должны выполняться условия $c_{n}=1, n$ - номер тестируемого элемента, остальные коэффициенты Фурье должны равняться нулю.

\section{4. Формирование базиса внутренних состояний}

Авторами [20] с помощью метода интегральных наложений установлена зависимость между пространственным напряженным и деформированным состоянием упругого трансверсально-изотропного конечного тела без полостей и определенными вспомогательными двумерными состояниями, компоненты которых зависят от двух координат $z$ и $y$ (переменных).

При установлении зависимости используется следующий прием. Упругое тело, напряженное состояние которого требуется изучить, рассматривается как часть некоторого бесконечного цилиндра с осью $\eta$, параллельной образующей цилиндpa. С телом связана система координат $r z \theta$. Меридианное сечение тела совпадает с плоскостью поперечного сечения бесконечного цилиндра с осями координат zy (направление $\eta \perp$ плоскости $z y$, ось $z$ общая для тела и цилиндра). Предполагается, что цилиндр находится в некотором двумерном напряженном состоянии не 
меняющемся вдоль образующей. Компоненты этого состояния $u_{y}^{p l}, u_{z}^{p l}, \sigma_{y}^{p l}$, $\sigma_{z}^{p l}, \sigma_{z y}^{p l}, \sigma_{\eta}^{p l}$ определяют плоскую деформацию цилиндра в плоскости zy. Эти же компоненты определяют напряженное состояние заданного упругого тела, так как оно является частью цилиндра. Для того чтобы получить пространственное напряженное состояние тела, рассматривается ряд цилиндров, отличающихся направлением образующей или углом поворота относительно оси $z$. Последовательно представляя тело вырезанным из каждого такого цилиндра при $m \rightarrow \infty$ ( $m$ - число цилиндров), образуется ряд напряженных состояний, суперпозиция которых и дает суммарное трехмерное состояние. Если напряженное состояние каждого цилиндра в процессе поворота не меняется, то трехмерное состояние тела будет осесимметричным, не зависящим от координаты $\theta$. Например, для компоненты вектора перемещения $w$ трехмерного состояния имеет место выражение

$$
w=\frac{1}{m} \sum_{k=1}^{m}\left(u_{z}^{p l}\right)_{k} .
$$

При переходе к пределу при $m \rightarrow \infty$ сумма заменяется интегралом. При последующей замене переменой интегрирования, окончательно связь между перемещениями осесимметричной деформации и плоской деформации соответствующего цилиндра имеет вид [20]

$$
u=\frac{1}{\pi} \int_{-r}^{r} \frac{u_{y}^{p l}}{r \sqrt{r^{2}-y^{2}}} d y, w=\frac{1}{\pi} \int_{-r}^{r} \frac{u_{z}^{p l}}{r \sqrt{r^{2}-y^{2}}} d y, v=0 .
$$

В работе [25] приведены выражения для напряжений осесимметричного состояния через напряжения плоского вспомогательного состояния.

Для построения поля перемещений плоских вспомогательных состояний воспользуемся системой многочленов $y^{\alpha} z^{\beta}$, которую можно поместить в любую позицию вектора перемещения $\mathbf{u}^{p l}(y, z)$, образуя некоторое допустимое состояние

$$
\mathbf{u}^{p l}=\left\{\left\{y^{\alpha} z^{\beta}, 0\right\},\left\{0, y^{\alpha} z^{\beta}\right\}\right\} .
$$

Перебор всевозможных вариантов в пределах $\alpha+\beta \leq n,(n=1,2,3 \ldots)$ позволяет получить множество плоских состояний. Далее, согласно (12), определяются компоненты вектора перемещения $\mathbf{u}(r, z)$ пространственного осесимметричного состояния и по цепочке (2), (3) определяются соответствующие тензоры деформаций и напряжений, образуя конечномерный базис (5).

\section{5. Решение задач для тел вращения}

Тестовая задача. Апробацию предложенной методики проведем на исследовании упругого состояния трансверсально-изотропного кругового в плане цилиндра из горной породы алевролита крупного темно-серого [22]. После процедуры обезразмеривания параметров задачи, аналогия которой представлена в работе [26], цилиндр занимает область $V=\{(z, r) \mid 0 \leq r \leq 1,-2 \leq z \leq 2\}$ и имеет упругие характеристики материала: $E_{z}=6.21 ; E_{r}=5.68 ; G_{r}=2.29 G_{z}=2.55 ; v_{z}=0.22$; $v_{r}=0.24$. 
В тестовой задаче предполагается подбор таких граничных условий, чтобы была возможность получения строгого решения. Зададим следующие неуравновешенные усилия на границе:

$$
\begin{gathered}
p_{r}=0 ; p_{z}=-1, S_{1} \mid z=-2,0 \leq r \leq 1 ; \\
p_{r}=0 ; p_{z}=2, S_{2} \mid z=2,0 \leq r \leq 1 ; \\
\mathbf{p}=0, S_{3} \mid r=1, \quad-2 \leq z \leq 2,
\end{gathered}
$$

и компоненты массовых сил $R=0, Z=-1 / 4$, последняя определена как разница главных векторов заданных сил, деленная на объем тела и направленная противоположно оси $z$.

После построения базиса внутренних состояний, процедуры его ортонормирования и исключения линейно зависимых элементов, базисный набор для компонент вектора перемещения представлен в таблице (показано 5 элементов).

Ортонормированный базисный набор компонент вектора перемещения

\begin{tabular}{|c|c|c|}
\hline & $u$ & $w$ \\
\hline$\xi_{1}$ & 0 & $0.263 z$ \\
\hline$\xi_{2}$ & $0.18528 r$ & $-0.10429 z$ \\
\hline$\xi_{3}$ & 0 & $0.11427 z^{2}$ \\
\hline$\xi_{4}$ & $0.15544 r z$ & $-0.04374 z^{2}$ \\
\hline$\xi_{5}$ & $-0.3983 r z$ & $0.32323 r^{2}+0.01121 z^{2}$ \\
\hline
\end{tabular}

Искомое состояние определено пятью членами ряда Фурье с соответствующими коэффициентами (11):

$$
c_{1}=0.79167, c_{2}=-0.31287, c_{3}=0.15235, c_{4}=-0.05832, c_{5}=0.01494 .
$$

Решение определяется выражениями (10), является строгим и имеет вид

$$
\begin{gathered}
u=-0.057971 r+0.0096618 r z ; w=0.0048309 r^{2}+0.241546 z+0.0201288 z^{2} ; \\
\sigma_{r r}=\sigma_{\theta \theta}=\sigma_{r \theta}=\sigma_{z \theta}=\sigma_{z r}=0 ; \sigma_{z z}=1.5+0.25 z ; R=0 ; Z=-0.25 .
\end{gathered}
$$

Расчетная задача для ичилиндра. Рассмотрим теперь для этого же цилиндра первую основную задачу механики с внешними усилиями, имитирующими однородное растяжение вдоль оси $z$ и неоднородное вдоль оси $r$ :

$$
\begin{gathered}
p_{r}=0 ; p_{z}=-1, S_{1} \mid z=-2,0 \leq r \leq 1 ; \\
p_{r}=0 ; p_{z}=1, S_{2} \mid z=2,0 \leq r \leq 1 ; \\
p_{r}=4-z^{2} ; p_{z}=0, S_{3} \mid r=1,-2 \leq z \leq 2
\end{gathered}
$$

и массовыми силами $\mathbf{X}=\left\{r^{2}, z\right\}$.

Сохранение той же геометрии и физических свойств материала, что и в тестовой задаче, позволяет использовать уже построенный ортонормированный базис. При решении использовался базис из 52 элементов. Приближенное решение имеет аналитический вид (приведем выражения для перемещений и массовых сил): 


$$
\begin{gathered}
u=\left[479558 r-1273.25 r^{3}-791.76 r^{5}+156.23 r^{7}-12190.3 r z^{2}+\right. \\
+665 r^{3} z^{2}-393.48 r^{5} z^{2}+1.54 r^{7} z^{2}-281.27 r z^{4}+40.083 r^{3} z^{4}+ \\
\left.+141.88 r^{5} z^{4}+124.87 r z^{6}-78.874 r^{3} z^{6}+3.105 r z^{5}\right] \cdot 10^{-5} \\
w=\left[10005.1 z+11670.4 r^{2} z+135.833 r^{4} z-88.783 r^{6} z+\right. \\
+8.0848 r^{8} z+100 z^{3}-936.646 r^{2} z^{3}+853.594 r^{4} z^{3}-6.3186 r^{6} z^{3}+ \\
\left.+153.371 z^{5}-191.719 r^{2} z^{5}-139 r^{4} z^{5}-24.56 z^{7}++41.8 r^{2} z^{7}\right] \cdot 10^{-5} ; \\
R=\left[220.8 r+1183.54 r^{3}-445.856 r^{5}-3.0348 r^{7}-4.8617 r z^{2}+\right. \\
\left.+136.936 r^{3} z^{2}-43.05 r^{5} z^{2}-28.8633 r z^{4}-35.207 r^{3} z^{4}+10.0678 r z^{6}\right] \cdot 10^{-3} ; \\
Z=\left[995.247 z+104.925 r^{2} z-70.404 r^{4} z-11.599 r^{6} z-21.853 z^{3}-\right. \\
\left.-102.278 r^{2} z^{3}+49.768 r^{4} z^{3}+25.2473 z^{5}+17.1775 r^{2} z^{5}-5.5309 z^{7}\right] \cdot 10^{-3} .
\end{gathered}
$$

Следует отметить, что сходимость в направлении поверхностных сил наблюдается быстрее, чем в направлении массовых сил. Поверхностные силы восстанавливаются с достаточной точностью уже при использовании 20 элементов базиса. Для достижения удовлетворительной точности в отношении массовых сил такого количества используемых элементов недостаточно.

При практической реализации приема решения первой основной задачи для цилиндра и его тестирования при различных видах функций заданных массовых сил наблюдалась следующая особенность: если область интегрирования $V$ симметрична относительно плоскости $z=0$, то сходимостью решения в части восстановления массовых сил обладают задачи с кососимметричной относительно этой плоскости компонентой массовых сил $Z$, например $Z=z^{n}, n=1,3,5 \ldots$. Это связано с видом базисных функций и, естественно, ограничивает круг рассматриваемых задач.

Рассмотрим теперь задачу для тела вращения неканонической формы (рис. 2) из того же материала, что и цилиндр. Исследуем распределение напряжений внутри области от действия массовых сил $\mathbf{X}=\left\{r^{2}, 0\right\}$ при условии, что след этих напряжений на границе равен нулю $\mathbf{p}=0$.

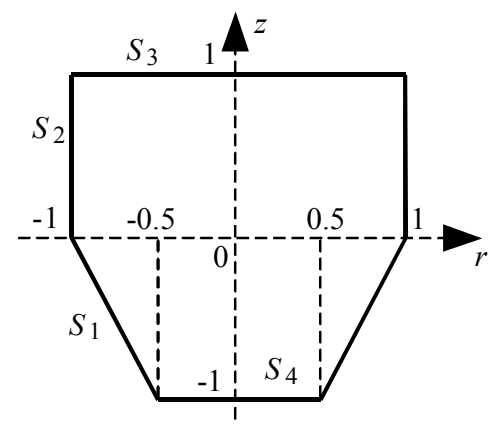

Рис. 2. Меридианное сечение тела вращения

Fig. 2. A meridian section of the body of revolution 
Сходимость решения сильно зависит от геометрии тела, поэтому для решения данной задачи потребовался базис уже из 70 элементов; коэффициенты Фурье приводить не будем. Проверка результата и оценка точности осуществляется сопоставлением заданных ГУ с восстановленными в результате решения. На рис. 3 показаны массовые силы на участках $S_{1}$ и $S_{2}$. На графиках заданные ( $\left.\begin{array}{llll}\text { l } & \text { l } & \text { l } & \text { l }\end{array}\right)$ и восстановленные (- массовые силы изображены в масштабе. Например, истинное значение $R$ на левом графике рис. $3, a$ равно значению на графике, умноженному на коэффициент $\kappa$.
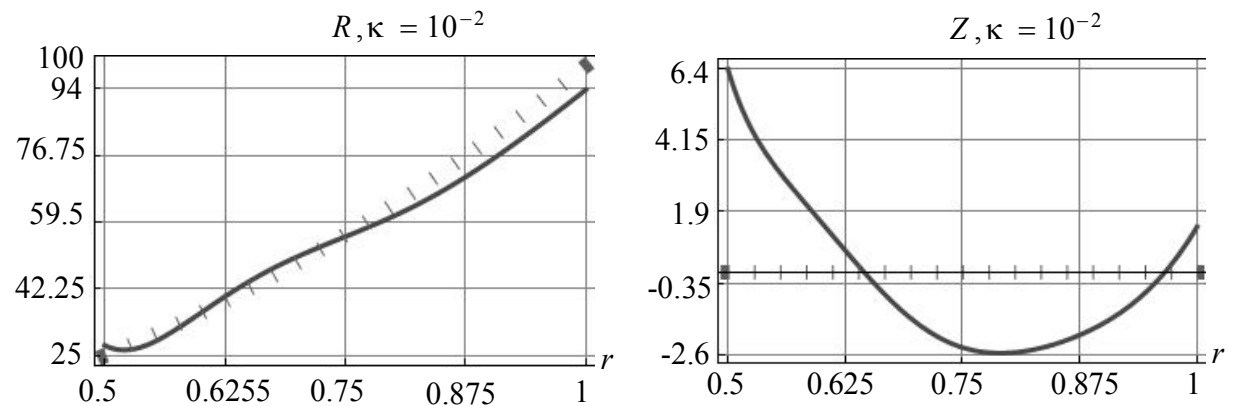

$a$
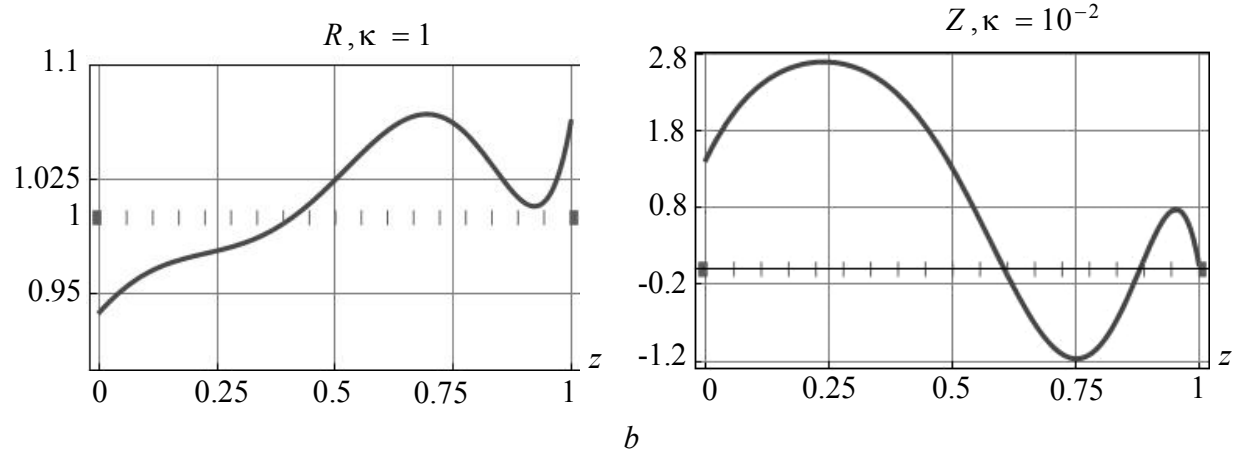

Рис. 3. Верификация массовых сил на участках границы:

$$
a \text { - на участке } S_{1}, b \text { - на участке } S_{2}
$$

Fig. 3. Verification of mass forces on the boundaries:

in the section (a) $S_{1}$ and (b) $S_{2}$

Как видно из графиков, восстановленные массовые силы совпадают с заданными в диапазоне определенной точности $( \pm 10 \%$ от значения заданной величины в любой точке на границе тела).

Компоненты вектора перемещения, имеющие полиномиальный вид, представлены в виде изолиний (в явном виде необозримы) (рис. 4). В силу осевой симметрии показана область $0 \leq r \leq 1,-1 \leq z \leq 1$. Истинное значение показанной величины равно соответствующему значению на изолиниях, умноженному на $\kappa$.

Полученные упругие поля удовлетворяют уравнениям (1) - (3), а также уравнениям совместности деформаций [23]. 


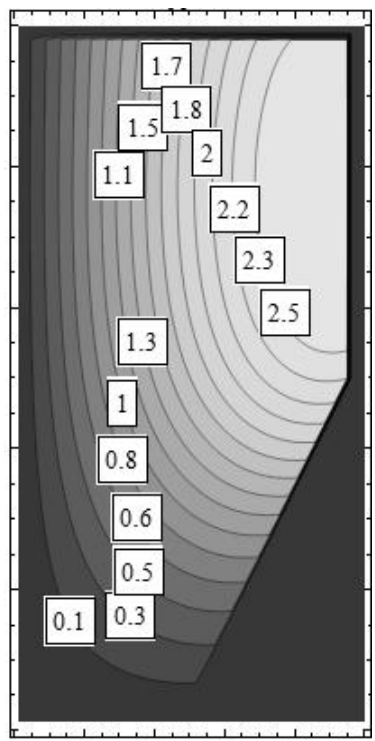

$a$

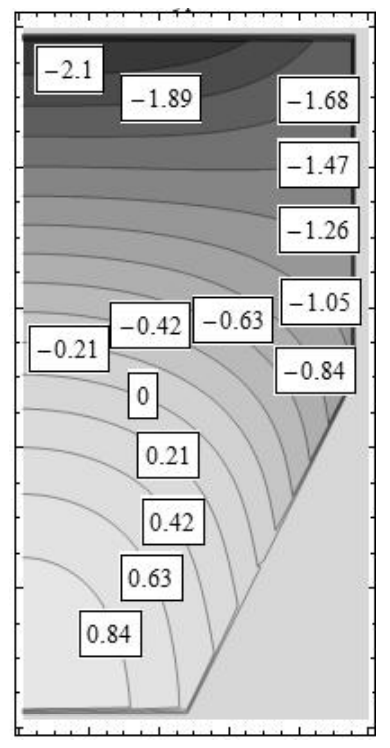

$b$

Рис. 4. Компоненты вектора перемещения:

$$
a-u, \kappa=10^{-2}, \sigma-w, \kappa=10^{-2}
$$

Fig. 4. Displacement vector components:

(a) $u, \kappa=10^{-2}$ and $(b) w, \kappa=10^{-2}$

\section{Заключение}

Таким образом, решение задач линейной теории упругости строится в виде рядов, но совершенно не опирается на общее или фундаментальное решение. Здесь задается зависимость вектора перемещения плоского вспомогательного состояния от координат $y^{\alpha} z^{\beta}$ и на его основе определяется вектор перемещения пространственного осесимметричного состояния. Для такого вектора по соотношению Коши определяется тензор деформаций, из закона Гука - тензор напряжений, из фундаментального соотношения Коши - усилия на поверхности тела, а из уравнения равновесия - массовые силы. То есть строится точное частное решение задачи, соответствующее заданной в каждой точке тела функции перемещения. Перебирая $\alpha+\beta \leq n(n=1,2,3 \ldots)$, строится множество точных частных решений задачи линейной теории упругости: векторы перемещения $\mathbf{u}_{k}$, тензоры деформаций $\boldsymbol{\varepsilon}_{k}$, тензоры напряжений $\boldsymbol{\sigma}_{k}$, векторы поверхностных сил $\mathbf{p}_{k}=\mathbf{n} \cdot \boldsymbol{\sigma}_{k}$, векторы массовых сил $\mathbf{X}_{k}$. Оставляя среди этих решений только линейно независимые и осуществляя их ортогонализацию в соответствии с соотношением (7), получаем базис, по которому соответствующие векторы или тензоры разлагаются в ряды с одинаковыми коэффициентами (11). Поэтому изложенный подход является более широким, чем подход, основанный на общих или фундаментальных решениях [25]. В данном случае используется процедура ортогонализации, определяемая соотношениями (7) и (9), позволяющая сразу строить решение задачи с заданными массовыми и поверхностными силами, а не строить полное решение как сумму 
частного решения задачи при действии массовых сил и общего решения при действии только поверхностных сил, как сделано, например, в работе [14].

Предложенная методика, однако, не является общей для любого класса рассматриваемых областей (односвязных и многосвязных) и вида заданных функций массовых сил. Скорость сходимости рядов зависит от граничных условий и условий внутри области, а также от геометрии тела.

Таким образом, метод граничных состояний показал свою эффективность в решении первой основной задачи теории упругости для трансверсальноизотропных тел вращения с массовыми силами. Результирующее упругое поле имеет численно-аналитический вид, что позволяет легко проводить анализ полученных характеристик напряженно-деформированного состояния.

\section{ЛИТЕРАТУРА}

1. Пикуль В.B. К аномальному деформированию твердых тел // Физическая мезомеханика. 2013. № 2. C. 90-100. DOI: 10.24411/1683-805X-2013-00019.

2. Голоскоков Д.П., Данилюк В.А. Моделирование напряженно-деформированного состояния упругих тел с помощью полиномов // Вестник Государственного университета морского и речного флота им. адмирала С.О. Макарова. 2013. № 1. С. 8-14.

3. Агаханов Э.К., Агаханов М.К. О моделировании действия объемных сил в упругоползучем теле // Изв. вузов. Северо-кавказский регион. Технические науки. 2005. № 1. C. 39-45.

4. Агаханов Э.К. О развитии комплексных методов решения задач механики деформируемого твердого тела // Вестник ДГТУ. Технические науки. 2013. № 2 (29). С. 39-45.

5. Агаханов Э.К. Решение задач механики деформируемого твердого тела с использованием фиктивных расчетных схем // Вестник ДГТУ. Технические науки. 2015. № 3(38). C. $8-15$.

6. Стружанов В.B. О решении краевых задач теории упругости методом ортогональных проекций // Математическое моделирование систем и процессов. 2004. № 12. С. 89-100.

7. Калантарлы H.M. Трещинообразование в круговом диске под действием объемных сил // Строительная механика инженерных конструкций и сооружений. 2014. № 6. С. 23-29.

8. Агаханов Э.К., Магомедэминов Н.С. Условия эквивалентности воздействий для перемещений // Вестник ДГТУ. Технические науки. 2007. №12. С. 27-28.

9. Пантелеев И.А., Полтавцева Е.В., Мубассарова В.А. Гаврилов В.А. Возмущение напряженно-деформированного состояния упругого полупространства шаровой неоднородностью упругих свойств при сдвиге в горизонтальной плоскости с учетом гравитационных сил // Вестник Пермского национального исследовательского политехнического университета. Механика. 2017. № 4. C. 138-153. DOI: 10.15593/perm.mech/2017.4.10.

10. Вестяк В.А., Тарлаковский Д.В. Нестационарное осесимметричное деформирование упругой толстостенной сферы под действием объемных сил // Прикладная механика и техническая физика. 2015. Т. 56. № 6. С. 59-69. DOI: 10.15372/PMTF20150608.

11. Матвеева А.Н. О влиянии силы тяжести на перемещения в упругопластической среде, ослабленной горизонтальной цилиндрической полостью // Материалы X Всероссийской конференции по механике деформируемого твердого тела, Самара, 2017. С. 75-77.

12. Фукалов А.А. Задачи об упругом равновесии составных толстостенных трансверсальноизотропных сфер, находящихся под действием массовых сил и внутреннего давления, и их приложения // XI Всероссийский съезд по фундаментальным проблемам теоретической и прикладной механики. Казань, 20-24 августа 2015. С. 3951-3953.

13. Ivanychev D.A., Levina E.Yu., Abdullakh L.S, Glazkova Yu.A. The method of boundary states in problems of torsion of anisotropic cylinders of finite length // Int. Trans. J. Engineering, Management, \& Applied Sciences \& Technologies. 2019. V. 10. No. 2. pp. 183-191. DOI: 10.14456/ITJEMAST.2019.18.

14. Левина Л.В, Кузьменко Н.В. Обратный метод эффективного анализа состояния упругого тела от массовых сил из класса непрерывных // XI Всероссийский съезд по фунда- 
ментальным проблемам теоретической и прикладной механики: сб. докл. Казань, 2015. C. 2276-2278.

15. Пеньков В.Б., Пеньков В.В., Викторов Д.В. Учет массовых сил в методе граничных состояний // Известия ТулГУ. Серия Математика. Механика. Информатика. 2005. Т. 11. Вып. 2. С. 94-100.

16. Penkov V.B., Ivanychev D.A., Novikova O.S., Levina L.V. An algorithm for full parametric solution of problems on the statics of orthotropic plates by the method of boundary states with perturbations // J. Physics: Conf. Series. 973. 2018. 012015. P. 1-10. DOI: 10.1088/17426596/973/1/012015.

17. Албагачиев А.Ю., Моисеенко А.М., Якобовская И.М., Зернов Е.В. Напряженнодеформированное состояние тонкой квадратной заготовки при ее осадке шероховатыми плитами // Вестник Томского государственного университета. Математика и механика. 2017. № 49. C. 75-80. DOI: 10.17223/19988621/49/7.

18. Пономарева M.A., Собко E.A., Якутенок B.A. Решение осесимметричных задач теории потенциала непрямым методом граничных элементов // Вестник Томского государственного университета. Математика и механика. 2015. № 5(37). С. 84-96.

19. Иванычев Д.А. Решение контактной задачи теории упругости для анизотропных тел вращения с массовыми силами // Вестник Пермского национального исследовательского политехнического университета. Механика. 2019. № 2. С. 49-62. DOI: 10.15593/ perm.mech/2019.2.05.

20. Александров А.Я., Соловьев Ю.И. Пространственные задачи теории упругости (применение методов теории функций комплексного переменного). М.: Наука, 1978. 464 c.

21. Пеньков В.Б., Пеньков В.В. Метод граничных состояний для решения задач линейной механики // Дальневосточный математический журнал. 2001. Т. 2. № 2. С. 115-137.

22. Лехницкий С.Г. Теория упругости анизотропного тела. 2-е изд. М.: Наука, 1977. 416 с.

23. Новацкий В. Теория упругости. М.: Мир, 1975. 872 с.

24. Саталкина Л.В. Наращивание базиса пространства состояний при жестких ограничениях к энергоемкости вычислений // Сборник тезисов докладов научной конференции студентов и аспирантов Липецкого государственного технического университета. 2007. С. $130-131$.

25. Иванычев Д.А. Метод граничных состояний в приложении к осесиметричным задачам для анизотропных тел // Вести высших учебных заведений Черноземья. 2014. № 1. C. 19-26.

26. Левина Л.В., Новикова О.С., Пеньков В.Б. Полнопараметрическое решение задачи теории упругости односвязного ограниченного тела // Вестник ЛГТУ. 2016. № 2(28). C. $16-24$.

Статья поступила 24.06.2019 г.

Ivanychev D.A. (2020) A METHOD OF BOUNDARY STATES IN A SOLUTION TO THE FIRST FUNDAMENTAL PROBLEM OF THE THEORY OF ANISOTROPIC ELASTICITY WITH MASS FORCES. Vestnik Tomskogo gosudarstvennogo universiteta. Matematika $i$ mekhanika [Tomsk State University Journal of Mathematics and Mechanics]. 66. pp. 96-111

DOI $10.17223 / 19988621 / 66 / 8$

Keywords: boundary state method, anisotropy, mass forces, boundary value problems, state space, first fundamental problem.

The purpose of this work is to determine the stress-strain state of the anisotropic bodies of revolution exposed to axisymmetric surface and mass forces. The problem is solved using the method of boundary states. A theory for the construction of space bases of the inner and boundary states conjugated with isomorphism is developed. Determination of the internal state is reduced to a study of isomorphic boundary state. The elastic state components are represented as Fourier series with quadrature coefficients. In the first fundamental problem of mechanics, determination of the elastic state is reduced to the solution of an infinite system of algebraic equations. 
A particularity of this solution is that the pattern of the determined elastic field satisfies both the conditions specified at the boundary and inside the body.

A rigorous solution to a test problem for a circular cylinder, as well as a solution to the problem with inhomogeneous boundary conditions is presented. An elastic field is found in the problem for the non-canonical body of revolution exposed to mass forces and zero boundary conditions. The explicit and indirect indicators of problem solution convergence and a graphical visualization of results are shown.

Financial support. The study was carried out with the financial support of the Russian Foundation for Basic Research and the Lipetsk Region in the framework of the research project No. 19-41-480003 "p_a".

Dmitriy A. IVANYCHEV (Candidate of Physics and Mathematics, Lipetsk State Technical University, Lipetsk, Russian Federation). E-mail: 1sivdmal@mail.ru

\section{REFERENCES}

1. Pikul' V.V. (2013) K anomal'nomu deformirovaniyu tverdykh tel [To the anomalous deformation of solids]. Fizicheskaya mezomekhanika - Physical Mesomechanics. 2. pp. 90-100. DOI: 10.24411/1683-805X-2013-00019.

2. Goloskokov D.P., Danilyuk V.A. (2013) Modelirovanie napryazhenno-deformirovannogo sostoyaniya uprugikh tel s pomoshch'yu polinomov [Modeling of a stress-strain state of elastic bodies using polynomials]. Vestnik gosudarstvennogo universiteta morskogo $i$ rechnogo flota imeni admirala S.O. Makarova. 1. pp. 8-14.

3. Agakhanov E.K., Agakhanov M.K. (2005) O modelirovanii deystviya ob"emnykh sil v uprugopolzuchem tele [On the modeling of the action of body forces in an elastic-creeping body]. Izvestiya vuzov. Severo-Kavkazskiy region. Tekhnicheskie nauki - University News. North-Caucasian Region. Technical Sciences Series. 1. pp. 39-45.

4. Agakhanov E.K. (2013) O razvitii kompleksnykh metodov resheniya zadach mekhaniki deformiruemogo tverdogo tela [On the development of complex methods for solving problems of mechanics of deformable solids]. Vestnik Dagestanskogo gosudarstvennogo tekhnicheskogo universiteta. Tekhnicheskie nauki - Herald of Dagestan State Technical University. Technical Sciences. 2(29). pp. 39-45.

5. Agakhanov E.K. (2015) Reshenie zadach mekhaniki deformiruemogo tverdogo tela $\mathrm{s}$ ispol'zovaniem fiktivnykh raschetnykh skhem [Solving problems of mechanics of deformable solids with the use of fictitious computational schemes]. Vestnik Dagestanskogo gosudarstvennogo tekhnicheskogo universiteta. Tekhnicheskie nauki - Herald of Dagestan State Technical University. Technical Sciences. 3(38). pp. 8-15.

6. Struzhanov V.V. (2004) O reshenii kraevykh zadach teorii uprugosti metodom ortogonal'nykh proektsiy [On the solution to boundary value problems of the theory of elasticity by the method of orthogonal projections]. Matematicheskoe modelirovanie sistem i protsessov. 12. pp. 89-100.

7. Kalantarly N.M. (2014) Treshchinoobrazovanie v krugovom diske pod deystviem ob"emnykh sil [Cracking in a circular disk under the action of volume forces]. Stroitel'naya mekhanika inzhenernykh konstruktsiy i sooruzheniy - Structural Mechanics of Engineering Constructions and Buildings. 6. pp. 23-29.

8. Agakhanov E.K., Magomedeminov N.S. (2007) Usloviya ekvivalentnosti vozdeystviy dlya peremeshcheniy [Equivalence conditions of impacts for displacements]. Vestnik Dagestanskogo gosudarstvennogo tekhnicheskogo universiteta. Tekhnicheskie nauki-Herald of Dagestan State Technical University. Technical Sciences. 12. pp. 27-28.

9. Panteleev I.A., Poltavtseva E.V., Mubassarova V.A., Gavrilov V.A. (2017) Vozmushchenie napryazhenno-deformirovannogo sostoyaniya uprugogo poluprostranstva sharovoy neodnorodnost'yu uprugikh svoystv pri sdvige $\mathrm{v}$ gorizontal'noy ploskosti s uchetom gravitatsionnykh sil [Perturbation of a stress-strain state of an elastic half-space by a spherical inhomogeneity of elastic properties under shear in horizontal plane taking account 
gravitational forces]. Vestnik Permskogo natsional'nogo issledovatel'skogo politekhnicheskogo universiteta. Mekhanika - PNRPU Mechanics Bulletin. 4. pp. 138-153. DOI: $10.15593 /$ perm.mech/2017.4.10.

10. Vestyak V.A., Tarlakovskiy D.V. (2015) Unsteady axisymmetric deformation of an elastic thick-walled sphere under the action of volume forces. Journal of Applied Mechanics and Technical Physics. 56(6). pp. 984-994. DOI: 10.1134/S0021894415060085.

11. Matveeva A.N. (2017) O vliyanii sily tyazhesti na peremeshcheniya v uprugoplasticheskoy srede, oslablennoy gorizontal'noy tsilindricheskoy polost'yu [On the effect of gravity on the motion in the elastoplastic medium weakened by a horizontal cylindrical cavity]. Proceedings of the X All-Russian Conference on Mechanics of Deformable Solids, Samara. pp. $75-77$.

12. Fukalov A.A. (2015) Zadachi ob uprugom ravnovesii sostavnykh tolstostennykh transversal'no-izotropnykh sfer, nakhodyashchikhsya pod deystviem massovykh sil i vnutrennego davleniya, i ikh prilozheniya [Problems on the elastic equilibrium of composite thick-walled transversally isotropic spheres under the action of mass forces and internal pressure, and their applications]. Proceedings of the XI All-Russian Congress on Basic Problems of Theoretical and Applied Mechanics, Kazan. pp. 3951-3953.

13. Ivanychev D.A., Levina E.Yu., Abdullakh L.S., Glazkova Yu.A. (2019) The method of boundary states in problems of torsion of anisotropic cylinders of finite length. International Transaction Journal of Engineering, Management, \& Applied Sciences \& Technologies. 10(2). pp. 183-191. DOI: 10.14456/ITJEMAST.2019.18.

14. Levina L.V., Kuz'menko N.V. (2015) Obratnyy metod effektivnogo analiza sostoyaniya uprugogo tela ot massovykh sil iz klassa nepreryvnykh [An inverse method of effective analysis of an elastic body state under mass forces from a class of continuous]. Proceedings of the XI All-Russian Congress on Basic Problems of Theoretical and Applied Mechanics, Kazan. pp. 2276-2278.

15. Pen'kov V.B., Pen'kov V.V., Viktorov D.V. (2005) Uchet massovykh sil v metode granichnykh sostoyaniy [The account for mass forces in the method of boundary states]. Izvestiya TulGU. Seriya Matematika. Mekhanika. Informatika. 11. pp. 94-100.

16. Penkov V.B., Ivanychev D.A., Novikova O.S., Levina L.V. (2018) An algorithm for full parametric solution of problems on the statics of orthotropic plates by the method of boundary states with perturbations. Journal of Physics: Conference Series. 973(012015). DOI: 10.1088/1742-6596/973/1/012015.

17. Albagachiev A.Yu., Moiseenko A.M., Yakobovskaya I.M., Zernov E.V. (2017) Napryazhenno-deformirovannoe sostoyanie tonkoy kvadratnoy zagotovki pri ee osadke sherokhovatymi plitami [A stress-strain state of a thin square workpiece during upsetting by rough plates]. Vestnik Tomskogo gosudarstvennogo universiteta. Matematika i mekhanikaTomsk State University Journal of Mathematics and Mechanics. 49. pp. 75-80. DOI: 10.17223/19988621/49/7.

18. Ponomareva M.A., Sobko E.A., Yakutenok V.A. (2015) Reshenie osesimmetrichnykh zadach teorii potentsiala nepryamym metodom granichnykh elementov [Solving axisymmetric potential problems using the indirect boundary element method]. Vestnik Tomskogo gosudarstvennogo universiteta. Matematika i mekhanika - Tomsk State University Journal of Mathematics and Mechanics. 5(37). pp. 84-96. DOI: 10.17223/19988621/37/8.

19. Ivanychev D.A. (2019) Reshenie kontaktnoy zadachi teorii uprugosti dlya anizotropnykh tel vrashcheniya s massovymi silami [Solution to a contact problem of the elasticity theory for anisotropic rotation bodies with mass forces]. Vestnik Permskogo natsional'nogo issledovatel'skogo politekhnicheskogo universiteta. Mekhanika - Bulletin of Perm National Research Polytechnic University. Mechanics. 2. pp. 49-62. DOI: 10.15593/ perm.mech/2019.2.05.

20. Aleksandrov A.Ya., Solov'ev Yu.I. (1978) Prostranstvennye zadachi teorii uprugosti (primenenie metodov teorii funktsiy kompleksnogo peremennogo) [Spatial problems of the theory of elasticity (application of the methods of the theory of functions of complex variable)]. Moscow: Nauka. 
21. Pen'kov V.B., Pen'kov V.V. (2001) Metod granichnykh sostoyaniy dlya resheniya zadach lineynoy mekhaniki [A method of boundary states for solving the problems of linear mechanics]. Dal'nevostochnyy matematicheskiy zhurnal. 2(2). pp. 115-137.

22. Lekhnitskiy S.G. (1977) Teoriya uprugosti anizotropnogo tela [The theory of elasticity for anisotropic body]. Moscow: Nauka.

23. Novatskiy V. (1975) Teoriya uprugosti [Theory of elasticity]. Moscow: Mir.

24. Satalkina L.V. (2007) Narashchivanie bazisa prostranstva sostoyaniy pri zhestkikh ogranicheniyakh $\mathrm{k}$ energoemkosti vychisleniy [Expansion of the basis of space of a state at severe limitations in the energy consumption of computations]. Theses of Reports at Scientific Conference of Lipetsk State Technical University for Students and Postgraduates. pp. 130-131.

25. Ivanychev D.A. (2014) Metod granichnykh sostoyaniy v prilozhenii k osesimetrichnym zadacham dlya anizotropnykh tel [A method of boundary states as applied to axisymmetric problems for anisotropic bodies]. Vesti vysshikh uchebnykh zavedeniy Chernozem'ya - News of Higher Educational Institutions of the Chernozem Region. 1. pp. 19-26.

26. Levina L.V., Novikova O.S., Pen'kov V.B. (2016) Polnoparametricheskoe reshenie zadachi teorii uprugosti odnosvyaznogo ogranichennogo tela [A full-parameter solution to the problem of the theory of elasticity of a simply connected bounded body]. Vestnik Lipetskogo gosudarstvennogo tekhnicheskogo universiteta - Bulletin of Lipetsk State Technical University. 2 (28). pp. 16-24.

Received: June 24, 2019 